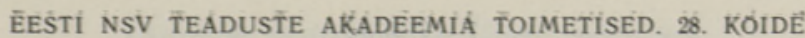
FOUSIKA - MATEMAATIKA. 1979, NR. 2

ИЗВЕСТИЯ АКАДЕМИИ НАУК ЭСТОНСКОП ССР. ТОМ 28 ФИЗИКА - МАТЕМАТИКА. 1979, № 2

\title{
LAGRANGE MULTIPLIERS IN LINEAR MULTISTAGE STOCHASTIC PROGRAMMING PROBLEMS
}

\author{
(Presented by N. Alumāe)
}

1. Let $(\Omega, \mathcal{F}, P)$ be a probability space with a nondecreasing family of $\sigma$-fields $\widetilde{F}_{1} \subseteq \mathcal{F}_{2} \subseteq \ldots \subseteq \mathscr{F}_{N} \subseteq \mathcal{F}_{\text {. }}$. Let $x=x(\omega): \Omega \rightarrow R^{n_{1}}=R^{n_{1}} \times \ldots \times R^{n_{N}}$ be a $\mathcal{F}$-measurable vector function. Denote by $X$ the set of functions $x(\omega)$ and let $M \subset X$ be the subset of all nonanticipative functions, i. e. $x \in M$ if $x(\omega)=\left\{x_{1}(\omega), \ldots, x_{N}(\omega)\right\}, \omega \in \Omega$ and $x_{k}(\omega)$ is $\mathcal{F}_{k}$-measurable, $k=$ $=1, \ldots, N$. Let $f: X \rightarrow R^{1}$ and $g(\omega, x(\omega))$ be a vector function, $g(\omega, \cdot)=$ $=y(\omega) \in Y$.

Let us consider the following $N$-stage stochastic programming problem (the exact formulation will be given below):

$$
\begin{aligned}
& f(x) \rightarrow \min , \\
& g(\omega, x(\omega)) \geqslant y_{0}(\omega), \quad y_{0}(\omega) \in Y, \\
& x \in M .
\end{aligned}
$$

In the statement of such an optimization problem it is frequently required that $x(\omega)$ must be a $P$-essentially bounded measurable function (for example, in addition to (2) and (3) it is required that $x(\omega) \in C$, where $C \subset R^{n}$ is a bounded set). It seems then natural to choose $X=Y=$ $=L^{\infty}\left(\Omega, \mathcal{F}, P ; R^{n}\right)$. The restriction (3) can also be treated as the functional restriction $\left[{ }^{1,2}\right]$, and under certain conditions the saddle-point theorem can be proved. The Lagrange multiplier which removes the constraint (3) belongs to the space $L^{1} \subset\left(L^{\infty}\right)^{*}$.

The space $L^{\infty}$ has the important property that the cone of his positive elements has nonempty interior. In the present note the problem is studied without this restriction, which makes it possible to handle the problem in the spaces $L^{p}, 1 \leqslant p<\infty$, for example. We consider the linear problem and we make the hypothesis that the minimum point exists. The necessary and sufficient conditions for the minimum in abstract linear programming problems are obtained in [3]. Our aim is to examine these conditions, taking into consideration the information constraint (3), typical in multistage stochastic programming problems.

2. Let $X, \tilde{Y}$ be the Banach spaces of certain $\mathcal{F}$-measurable functions and let $X^{*}, \tilde{Y}^{*}$ be their conjugate spaces. Denote by $K_{\tilde{Y}}$ the closed convex cone of positive elements in $\bar{Y}$ and let $K_{\tilde{Y}}^{*}$ be the conjugate cone. Let $E_{k} x=E^{\mathcal{F}^{k} x}$ be the conditional expectation of $x(\omega) \in X$ with respect to $\mathcal{F}_{k}$. Suppose that $E_{k}: X \rightarrow X$ is the continuous linear operator (as it is 
for $\left.X=L^{p}\left(\Omega, \mathcal{F}, P ; R^{n}\right)\right)$ and denote $A_{1} x=\left(x_{1}-E_{1} x_{1}, \ldots, X_{N}-E_{N} x_{N}\right)$. Let $A_{2}: X \rightarrow \tilde{Y}$ be the continuous linear operator and $A x=\left(A_{1} x, A_{2} x\right)$. Denote $Y=X \times \bar{Y}, K_{Y}=\{0\} \times K_{\bar{Y}}, 0 \in X$. Let $y_{0}=\left(0, \tilde{y}_{0}\right) \in Y$ be fixed and let $f \in X^{*}$.

The following extremal problem will be studied:

$$
\begin{aligned}
& f(x) \rightarrow \min , \\
& A x \geqslant y_{0} .
\end{aligned}
$$

The inequality $A x \geqslant y_{0}$ means that $A_{1} x=0$ and $A_{2} x \geqslant \tilde{y}_{0}$. The constraint $A_{1} x=0$ is typical in the sequential optimization problems. This emphasizes the fact that the decisions at every stage must depend only upon the past observations and not on the future ones.

3. Let $x_{0} \in X$ be the minimum point. Let us fix the element $z_{0} \in-K_{Y}$ and denote (cf. [ $\left.\left.{ }^{3}\right]\right) K\left(z_{0}\right)=\left\{\lambda z_{0}: \lambda \geqslant 0\right\}+K_{Y}=\left\{\lambda z_{0}:-\infty<\lambda<\infty\right\}+K_{Y}$ and $P\left(z_{0}\right)=\left\{y^{*}: y^{*} \in K_{Y^{*}}, y^{*}\left(z_{0}\right)=0\right\}$. Let $\operatorname{cl} B$ be the strong closure of the set $B$.

Lemm a 1. $P\left(z_{0}\right)=\left(\operatorname{cl} K\left(z_{0}\right)\right)^{*}$.

Let $y_{1}=y+\lambda z_{0} \in K\left(z_{0}\right)$, where $y \in K_{Y}, \lambda \geqslant 0, z_{0} \in-K_{Y}$. If $y^{*} \in P\left(z_{0}\right)$, then $y^{*}\left(y_{1}\right)=y^{*}(y)+\lambda y^{*}\left(z_{0}\right)=y^{*}(y) \geqslant 0$, i. e. $y^{*} \in\left(K\left(z_{0}\right)\right)^{*}$. Conversely, if $y^{*}\left(y_{1}\right)=y^{*}(y)+\lambda y^{*}\left(z_{0}\right) \geqslant 0$ for all $\lambda \in R^{1}$, then $y^{*}\left(z_{0}\right)=0$, i. e. $y^{*} \in P\left(z_{0}\right)$. It remains to notice that $\left(K\left(z_{0}\right)\right)^{*}=\left(\operatorname{cl} K\left(z_{0}\right)\right)^{*}$.

Let $A^{*}$ be the adjoint of $A$. Let us introduce the following conditions.

$M\left(z_{0}\right): A^{-1}\left(\operatorname{cl} K\left(z_{0}\right)\right)=\operatorname{cl}\left(A^{-1} K\left(z_{0}\right)\right)$;

$N\left(z_{0}\right): A^{*} P\left(z_{0}\right)$ is weakly ${ }^{*}$ closed in $X^{*}$;

$F\left(z_{0}\right):\left(A^{-1} K\left(z_{0}\right)\right)^{*}=A^{*}\left(K\left(z_{0}\right)\right)^{*}$.

The conditions $M\left(z_{0}\right)$ and $N\left(z_{0}\right)$ were introduced in $\left[{ }^{3}\right]$. It was shown that when they are satisfied, then $x_{0}$ is the minimum point iff there exists $y_{0}{ }^{*} \in Y^{*}$ such that $\left(x_{0}, y_{0}{ }^{*}\right)$ is the saddle-point of the Lagrangian $H\left(x, y^{*}\right)=f(x)-y^{*}\left(A x-y_{0}\right)$. If only $M\left(z_{0}\right)$ holds, then only the generalized saddle-point theorem can be proved [ $\left.{ }^{3}\right]$.

Frequently $\left[{ }^{4}\right]$ the necessary conditions for the minimum, are proved under the condition $F\left(z_{0}\right)$ (the Farkas' condition). We shall prove that the conditions $M\left(z_{0}\right)$ and $N\left(z_{0}\right)$ are equivalent to the condition $F\left(z_{0}\right)$. The importance of dividing the Farkas' condition into two parts lies in the fact that this enables to separate the case when only the generalized saddle-point theorem is valid.

Theorem 1. $F\left(z_{0}\right)$ is satisfied iff $M\left(z_{0}\right)$ and $N\left(z_{0}\right)$ are satisfied.

Let $M\left(z_{0}\right)$ and $N\left(z_{0}\right)$ be satisfied. By $M\left(z_{0}\right)$ we have $\left(A^{-1} K\left(z_{0}\right)\right)^{*}=$ $=\left(\operatorname{cl} A^{-1} K\left(z_{0}\right)\right)^{*}=\left(A^{-1} \operatorname{cl} K\left(z_{0}\right)\right)^{*}$. From Lemma $1 A^{*}\left(\operatorname{cl} K\left(z_{0}\right)\right)^{*}=$ $=A^{*} P\left(z_{0}\right)$ and by $N\left(z_{0}\right)$ this cone is weakly* closed. Therefore ([5] p. 118) $\left(A^{-1} \operatorname{cl} K\left(z_{0}\right)\right)^{*}=A^{*}\left(\operatorname{cl} K\left(z_{0}\right)\right)^{*}$. Thus we have $\left(A^{-1} K\left(z_{0}\right)\right)^{*}=$ $=A^{*}\left(\mathrm{cl} K\left(z_{0}\right)\right)^{*}=A^{*}\left(K\left(z_{0}\right)\right)^{*}$, i. e. the condition $F\left(z_{0}\right)$ holds.

Now let $F\left(z_{0}\right)$ be satisfied. $\left(A^{-1} K\left(z_{0}\right)\right)^{*}$ is weakly* closed and therefore the cone $A^{*}\left(K\left(z_{0}\right)\right)^{*}=A^{*} P\left(z_{0}\right)$ is also weakly* closed, i. e. $N\left(z_{0}\right)$ holds. From the preceding paragraph and $F\left(z_{0}\right)$ we have

$$
\begin{gathered}
\left(\operatorname{cl} A^{-1} K\left(z_{0}\right)\right)^{*}=\left(A^{-1} K\left(z_{0}\right)\right)^{*}=A^{*}\left(K\left(z_{0}\right)\right)^{*}=A^{*}\left(\operatorname{cl} K\left(z_{0}\right)\right)^{*}= \\
=\left(A^{-1} \operatorname{cl} K\left(z_{0}\right)\right)^{*} .
\end{gathered}
$$

Suppose that $M\left(z_{0}\right)$ is not satisfied, i. e. $A^{-1} \mathrm{cl} K\left(z_{0}\right) \supset \mathrm{cl} A^{-1} K\left(z_{0}\right)$. Then exists $x_{1} \in A^{-1} \mathrm{cl} K\left(z_{0}\right)$ such that $x_{1} \equiv \mathrm{cl} A^{-1} K\left(z_{0}\right)$. This element $x_{1}$ can 
be strictly separated from the cone $\operatorname{cl} A^{-1} K\left(z_{0}\right)$, i. e. there exists $g \in X^{*}$ such that $g(x) \geqslant 0>g\left(x_{1}\right)$, for all $x \in \mathrm{cl} A^{-1} K\left(z_{0}\right)$, contrary to $(6)$.

4. Consider now the condition $M\left(z_{0}\right)$ for the operator $A=\left(A_{1}, A_{2}\right)$. If $z_{0}=\left(0, \tilde{z}_{0}\right), \quad 0 \in X, \quad \tilde{z}_{0} \in-K_{\tilde{Y}}, \quad$ then $K\left(z_{0}\right)=\{0\} \times \widetilde{K}\left(\tilde{z}_{0}\right), \quad \widetilde{K}\left(\tilde{z}_{0}\right)=$ $\doteq\left\{\lambda \tilde{z}_{0}: \lambda \geqslant 0\right\}+K_{\tilde{Y}}$ and $\operatorname{cl} K\left(z_{0}\right)=\{0\} \times \operatorname{cl} \tilde{K}\left(\tilde{z}_{0}\right)$. Denote by int $B$ and ri $B$ interior and relative interior $\left.{ }^{[6}\right]$ of the set $B$ and let $A_{1} X=\bar{X} \subset X$.

Le $\mathrm{mma}$ 2. Let $K_{1}$ and $K_{2}$ be convex cones in $X$ and let

$$
K_{1} \cap \text { ri } K_{2} \neq \varnothing, \quad \text { cl } K_{2}+\text { ri } K_{2}=\text { ri } K_{2} \text {. }
$$

Then

$$
\operatorname{cl}\left(K_{1} \cap K_{2}\right)=\operatorname{cl} K_{1} \cap \mathrm{cl} K_{2} .
$$

Note $\left[{ }^{4}\right]$ that (8) (and also (7)) are satisfied when $K_{1} \cap$ int $K_{2} \neq \varnothing$. The lemma can be proved in the same manner.

Ex a mple. Let $K_{2}$ be the cone of polynomials of degree $\leqslant n$ in $L^{2}$. Then int $K_{2}=\varnothing$ but ri $K_{2}=K_{2}$ and cl $K_{2}+$ ri $K_{2}=$ ri $K_{2}$. If $K_{2}$ is the cone of all polynomials in $L^{2}$, then int $K_{2}=\varnothing$, ri $K_{2}=K_{2}$ but $\mathrm{cl} K_{2}=L^{2}$ and $\mathrm{cl} K_{2}+$ ri $K_{2} \neq$ ri $K_{2}$.

Theorem 2. Let $M\left(\tilde{z}_{0}\right)$ be satisfied for $A_{2}$. If the convex cones $K_{1}=$ $=\operatorname{ker} A_{1}=\left\{x \in X: A_{1} x=0\right\}$ and $K_{2}=A_{2}{ }^{-1} \mathrm{cl} \widetilde{K}\left(\tilde{z}_{0}\right)$ satisfy (7), then $M\left(z_{0}\right)$ holds for $A$.

Clearly, $A^{-1} \operatorname{cl} K\left(z_{0}\right)=\operatorname{ker} A_{1} \cap A_{2}{ }^{-1} \operatorname{cl} \widetilde{K}\left(\tilde{z}_{0}\right)$ and $\operatorname{ker} A_{1}=\operatorname{cl} \operatorname{ker} A_{1}$. By $M\left(\tilde{z}_{0}\right)$ and Lemma $2 \operatorname{cl} A^{-1} K\left(z_{0}\right)=\operatorname{cl}\left[\operatorname{ker} A_{1} \cap A_{2}^{-1} \widetilde{K}\left(\tilde{z}_{0}\right)\right]=$ $=\operatorname{ker} A_{1} \cap A_{2}{ }^{-1} \mathrm{cl} \tilde{K}\left(\tilde{z}_{0}\right)$.

Consider now the condition $N\left(z_{0}\right)$. Note that $A^{*}=\left(A_{1}{ }^{*}, A_{2}{ }^{*}\right)$ and $K_{Y}{ }^{*}=\bar{X}^{*} \times K_{\tilde{Y}^{*}}{ }^{*}$. Therefore $P\left(z_{0}\right)=\bar{X}^{*} \times P\left(\tilde{z}_{0}\right)$ and $A^{*} P\left(z_{0}\right)=A_{1}{ }^{*} \bar{X}^{*}+$ $+A_{2}{ }^{*} P\left(\tilde{z}_{0}\right)$.

The range of $A_{1}$ is closed and therefore $A_{1}{ }^{*} \bar{X}^{*}$ is weakly ${ }^{*}$ closed. If $N\left(\tilde{z}_{0}\right)$ holds for $A_{2}$, then the second term is also weakly* closed, but the sum of two weakly* closed sets need not necessarily be weakly* closed.

Lemma 3. Let $Z$ be a Banach space, $Z_{1} \subset Z$ - its subspace and $K \subset Z$ - the cone with nonempty interior. Let $Z_{1} \cap$ int $K \neq \varnothing$. Then $Z_{1}+K=Z$.

Let $x_{0} \in Z_{1} \cap$ int $K$ and $S \subset Z$ be such sphere with the centre at zero that $x_{0}+S \subset K$. Then $S=\left(x_{0}+S\right)-x_{0} \subset K-Z_{1}=K+Z_{1}$. For every $\lambda \geqslant 0$ $\lambda S \subseteq K+Z_{1}$, therefore, $K+Z_{1}=Z$.

Now it is easy to state the following sufficient condition for $N\left(z_{0}\right)$ : if $A_{1}{ }^{*} \bar{X}^{*} \cap$ int $A_{2}{ }^{*} P\left(z_{0}\right) \neq \varnothing$, then $N\left(z_{0}\right)$ holds. By Lemma 3 it is immediate that $A_{1}{ }^{*} \bar{X}^{*}+A_{2}{ }^{*} P\left(\tilde{z}_{0}\right)=\bar{X}^{*} X Y^{*}$ and $N\left(z_{0}\right)$ holds.

Note that if for the operator $A_{2}$ some Slater-type assumption holds, i. e.

$$
A_{2}\left(\operatorname{ker} A_{1}\right) \cap \text { int } K\left(\tilde{z}_{0}\right) \neq \varnothing,
$$

then $F\left(z_{0}\right)$ holds $\left[{ }^{4}\right]$ and by Theorem 1 also $M\left(z_{0}\right)$ and $N\left(z_{0}\right)$.

\section{REFERENCES}

1. Rock a fell a r, R. T., We ts, R. J.-B., J. Math. Econ., 2, 349-370 (1975).

2. Evstigneev, I. V., Lecture notes in economics and mathematical systems, 133, Berlin, Springer, 1976, p. 34-48.

3. Л е в н В. Л., В сб.: Исследования по математическому программированию, М., «Наука», 1968, с. $159-197$. 
4. Kurcyusz, S., J. Optimiz. Theory and Appl., 20, № 1, 81-110 (1976).

5. Эр р оу К. Дж., Г ур в иц Л., Уд з а в а Х., Исследования по линейному и нелинейному программированию, М., Изд-во иностр. лит., 1962.

6. Р ок а фелл а р Р., Выпуклый анализ, М., «Мир», 1973.

Academy of Sciences of the Estonian SSR, Institute of Cybernetics

Received May 5, 1978

T. TOBIAS

\section{LAGRANGE'I KORDAJAD LINEAARSETES MITMEETAPILISTES STOHHASTILISE PROGRAMMEERIMISE OLESANNETES}

Lõpmatumõõtmelise programmeerimise meetodite abil on tuletatud tarvilikud tingimused lineaarsetele mitmeetapilistele stohhastilise programmeerimise ülesannetele ning uuritud informatsiooniliste kitsenduste mõju neile.

\section{T. ТОБИАС}

\section{МНОЖИТЕЛИ ЛАГРАНЖА В ЛИНЕИНЫХ МНОГОЭТАПНЫХ ЗАДАЧАХ СТОХАСТИЧЕСКОГО ПРОГРАММИРОВАНИЯ}

Методами бесконечномерного программирования выводятся необходимые условия экстремума в линейных многоэтапных задачах стохастического программирования. Рассматриваются пространства, где конус положительных элементов не имеет внутренних точек. Исследуется влияние информационных ограничений на эти условия и выделяется случай,' когда множители Лагранжа существуют в обобщенном смысле. 